Acta Crystallographica Section D

Biological Crystallography

ISSN 0907-4449

\section{Jiansheng Jiang, ${ }^{a}$ Enrique Abola ${ }^{a}$ and Joel L. Sussman ${ }^{\mathrm{a}, \mathrm{b} *}+$}

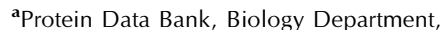
Building 463, Brookhaven National Laboratory, Upton, NY 11973-5000, USA, and 'bepartment of Structural Biology, Weizmann Institute of Science, Rehovot 76100, Israel

† Head, Protein Data Bank.

Correspondence e-mail: sussman@pdb.pdb.bnl.gov

Table 1

PDB structure-factor (SF) submission.

\begin{tabular}{lcl}
\hline Year & $\begin{array}{l}\text { Number of X-ray } \\
\text { structure submissions }\end{array}$ & $\begin{array}{l}\text { Number of SF } \\
\text { submissions (\%) }\end{array}$ \\
\hline 1994 & 804 & $205(25.0)$ \\
1995 & 963 & $343(36.0)$ \\
1996 & 1124 & $546(49.0)$ \\
1997 & 1484 & $932(62.8)$ \\
$1998 \dagger$ & 1616 & $868(53.7)$ \\
Total & 5991 & $2894(48.3)$ \\
\hline
\end{tabular}

$\dagger$ As of November 241998

\title{
Deposition of structure factors at the Protein Data Bank
}

The Protein Data Bank (PDB) has long made available the experimental data which were used to determine the three-dimensional structures in the database. In recent years more and more depositors and users of the PDB have come to appreciate the importance of reliable access to such fundamental data. The deposition of the experimental data, along with the coordinates is essential for the following reasons.

(i) Rigorous validation of the structuredetermination results can only be carried out using both atomic parameters and experimental structure-factor amplitudes.

(ii) Archiving of this data will ensure their preservation and continued accessibility.

Whether or not to require that the experimental data be deposited concomitantly with the structure data has been hotly discussed recently in the scientific press [Baker, Blundell, Vijayan, Dodson, Dodson, Gilliland \& Sussman (1996). Nature (London), 379, 202] and on the internet (EBI/MSD Draft Consultative Document for Deposition of Structure Factors http://croma.ebi.ac.uk/msd/Policy/sf.html).

At present more than $50 \%$ of the X-ray diffraction submissions are being deposited with their associated structure factors (see Table 1), compared with $25 \%$ four years ago. This increase is probably partly due to the ease of uploading the files via our WWW-based submission tool, AutoDep, and the fact that this tool is available both in the USA at BNL (PDB deposition site at http://www.pdb. bnl.gov) and in Europe at the EBI (EBI deposition site at http://www2.ebi.ac.uk/pdb). The PDB strongly encourages all researchers to deposit their structure factors at the time of coordinate submission. Furthermore, we actively encourage journals to require their submission as a prerequisite for publication [Sussman (1996). Protein Data Bank Quart. Newslett. No. 75 , p. 1, at ftp://pdb.pdb.bnl.gov/ newsletter/newsletter96jan/newslttr.txt].

In order to facilitate the use of deposited structure factors, we at the PDB, together with a number of macromolecular crystallographers and the IUCr Working Group on Macromolecular CIF, developed a standard interchange format for structure factors [PDB Structure Factor mmCIF at ftp://pdb. pdb.bnl.gov/pub/pdb/structure_factors/cifSF_ dictionary; Protein Data Bank Quart. Newslett. No. 74, p. 1 (1995), at ftp://pdb.pdb.bnl.gov/ newsletter/newsletter95oct/newslttr.tx]. This standard is the mmCIF format, i.e. the IUCrdeveloped Macromolecular Crystallographic Information File. It was chosen for its simplicity of design and for being clearly selfdefining. The format is also easy to expand, as new crystallographic experimental methods or concepts are developed, by simply adding additional tokens. The entire mmCIF crystallographic dictionary (http://ndb.rutgers.edu/ $\mathrm{NDB} /$ mmcif) has recently been ratified by the IUCr's COMCIFS committee.

The PDB has written a program to quickly and easily convert structure factors, as output by the most frequently used crystallographic programs, into the mmCIF format. This tool, which also converts binary $C C P 4 \mathrm{MTZ}$ files, will be accessible through the AutoDep program following final testing. MTZ files, which are useful in individual laboratories, are not appropriate for archival purposes. This is because particular groups arbitrarily attach different labels to the MTZ columns.

During the past year, the PDB has converted virtually all the old structure-factor files to this standard format and is keeping up-to-date on all new submissions. As of November 1998, there $\sim 2000$ structure-factor files released in the structure-factor mmCIF format (PDB mmCIF structure-factor files can be found at ftp://pdb. pdb.bnl.gov/pub/pdb/structure_factors/CIF_ format), with an additional $\sim 1300$ 'on-hold' for up to four years according to the IUCr policy (see IUCr deposition policy at http://www.iucr.org/ iucr-top/journals/acta/actad_notes.html). The structure factors are also available through the PDB's WWW-based 3DB Browser (http:// www.pdb.bnl.gov/pdb-bin/pdbmain). This can be seen on the browser's atlas page for each structure.

The ready availability of structure-factor files in a standard format has made it possible for any scientist to validate a structure in the PDB versus its experimentally observed data. There are now some excellent tools available for this, such as SFCHECK (http://www.iucr.org/iucrtop/comm/ccom/School96/pdf/sw.pdf) and the Uppsala Electron Density Server (http://alpha2. bmc.uu.se/valid/density/form1.html). The PDB has also observed that one of the most popular uses for these stored structure factors is for the crystallographer who did the experiment to be able to retrieve his/her own data which have been misplaced in their laboratory. 